DOI: $10.33947 / 1982-3282-v 15 n 3-4-4667$

\title{
SAÚDE MENTAL DE PESSOAS EM SITUAÇÃO DE RUA: COMPORTAMENTOS E VULNERABILIDADES NO CONTEXTO URBANO
}

\section{MENTAL HEALTH OF PEOPLE IN STREET SITUATION: BEHAVIORS AND VULNERABILITIES IN THE URBAN CONTEXT}

\section{SALUD MENTAL DE LAS PERSONAS EN SITUACIÓN DE CALLE: COMPORTAMIENTOS Y VULNERABILIDADES EN EL CONTEXTO URBANO}

\begin{abstract}
Felicialle Pereira da Silva ${ }^{1}$, Iracema da Silva Frazão², Raphael Alves da Silva ${ }^{3}$, Antônio Sávio Inácio, Henry Johnson Passos de Oliveira ${ }^{5}$, Selene Cordeiro Vasconcelos ${ }^{6}$, Murilo Duarte da Costa Lima ${ }^{7}$
\end{abstract}

Submetido em: 22/02/2021

Aceito em: 28/07/2021

\section{RESUMO}

Introdução: A população em situação de rua está exposta aos diversos riscos para a saúde, como a dificuldade em acessar os serviços de saúde. Objetivo: analisar a saúde mental de pessoas em situação de rua, comportamentos e vulnerabilidades no contexto urbano. Método: estudo exploratório, transversal e quantitativo. A amostra foi constituída de 274 pessoas maiores de 18 anos. Resultados: identificamos que 175 participantes apresentaram padrão de uso abusivo de drogas, dos quais $(20 \%)$ com indicação de tratamento intensivo para o uso do álcool. Discussão: 0 consumo de drogas é um fenômeno presente neste modo de vida que inclui processos adaptativos, quer seja pelo uso individual, ou em grupos, buscando assim aliviar as adversidades vivenciadas nas ruas. Conclusão: a saúde mental da população de rua continua fragilizada pelo prejuízo do atendimento às necessidades básicas, a dificuldade de acesso aos serviços de saúde e dos comportamentos de risco da vida nas ruas.

DESCRITORES: Pessoas em situação de rua; Saúde mental; Vulnerabilidade em Saúde; Área Urbana.

\section{ABSTRACT}

Introduction: The homeless population is exposed to several health risks, such as the difficulty in accessing health services. Objective: to analyze the mental health of people on the street, behaviors and vulnerabilities in the urban

\footnotetext{
${ }^{1}$ Enfermeira doutora em Neuropsiquiatria e ciências do comportamento- UFPE, professora do curso de enfermagem do Centro Universitário Mauricio de Nassau- UNINASSAU e professora adjunta da Universidade de Pernambuco- UPE, Departamento de Enfermagem. Recife, Pernambuco, Brasil. https://orcid.org/0000-0002-2805-7506, cialle@hotmail.com

${ }^{2}$ Enfermeira, Doutorado em Serviço Socia-UFPE, professor associado III da Universidade Federal de Pernambuco. Departamento de Enfermagem. Recife, Pernambuco, Brasil. https://orcid.org/0000-0002-4690-3753, isfrazao@gmail.com

${ }^{3}$ Acadêmico de Enfermagem. Centro Universitário Mauricio de Nassau- UNINASSAU, Departamento de Enfermagem. Recife, Pernambuco, Brasil. https://orcid.org/0000-0003-2555-909X, raphaelalves770@hotmail.com.

${ }^{4}$ Enfermeiro, Mestrando em neuropsiquiatria e ciências do comportamento-UFPE. Recife, Pernambuco, Brasil. https://orcid. org/0000-0001-5145-1803. savio08ignacio@gmail.com

${ }^{5}$ Enfermeiro, Residente em Saúde Coletiva pelo Instituto Aggeu Magalhães- FIOCRUZ-PE, departamento de saúde coletiva. Recife, Pernambuco, Brasil. https://orcid.org/0000-0001-8545-8640, henry123johnson@hotmail.com.

6 Enfermeira, doutora em Neuropsiquiatria e ciências do comportamento- UFPE, Professora adjunta da Universidade Federal da Paraíba-UFPB, João pessoa, Paraíba, Brasil. https://orcid.org/0000-0002-8828-1251. selumares@gmail.com 7 Médico, doutor em medicina. Universitat de Barcelona, Espanha, professor titular. do Departamento de Neuropsiquiatria do Centro de Ciências da Saúde da Universidade Federal de Pernambuco. Recife, Pernambuco, Brasil. https://orcid.org/00000002-1083-5517. murilocostalima@ig.com.br
} 
context. Method: exploratory, cross-sectional and quantitative study. The sample consisted of 274 people over 18 years of age. Results: we identified that 175 participants showed a pattern of drug abuse, of which (20\%) indicated intensive treatment for alcohol use. Discussion: Drug use is a phenomenon present in this way of life that includes adaptive processes, whether by individual use, or in groups, thus seeking to alleviate the adversities experienced on the streets. Conclusion: the mental health of the street population remains weakened by the loss of meeting basic needs, the difficulty of accessing health services and the risky behaviors of life on the streets.

DESCRIPTORS: Homeless people; Mental health; Health Vulnerability; Urban area.

\section{RESUMEN}

Introducción: La población sin hogar está expuesta a varios riesgos para la salud, como la dificultad para acceder a los servicios de salud. Objetivo: analizar la salud mental de las personas en la calle, comportamientos y vulnerabilidades en el contexto urbano. Método: estudio exploratorio, transversal y cuantitativo. La muestra estuvo formada por 274 personas mayores de 18 años. Resultados: identificamos que 175 participantes presentaron patrón de abuso de drogas, de los cuales (20\%) indicaron tratamiento intensivo por consumo de alcohol. Discusión: El consumo de drogas es un fenómeno presente en esta forma de vida que incluye procesos adaptativos, ya sea por consumo individual o grupal, buscando así paliar las adversidades vividas en las calles. Conclusión: la salud mental de la población de la calle sigue debilitada por la pérdida de la satisfacción de las necesidades básicas, la dificultad para acceder a los servicios de salud y las conductas de riesgo de la vida en la calle.

DESCRIPTORES: Personas sin hogar; Salud mental; Vulnerabilidad sanitaria; Área urbana. 


\section{INTRODUÇÃO}

A vida nas ruas não é um problema recente, tão pouco desconhecido pela sociedade, ou pelos poderes midiáticos e políticos. Trata-se de uma grave questão que vem acumulando desafios ao longo do tempo, sobretudo no espaço urbano ${ }^{1}$. Há um crescente número de pessoas que vivem abaixo da linha da pobreza, como indigentes, excluídos das estruturas convencionais da sociedade, vivendo em situação de rua. Este grupo de indivíduos vivenciam a falta de habitação regular, possuem vínculos familiares rompidos ou fragilizados, além da exposição aos diversos fatores de risco, como por exemplo, a dificuldade em acessar aos serviços de saúde².

Atualmente, o Brasil não dispõe de dados atualizados sobre o contingente da população de rua, uma vez que o último censo nacional para esta população foi realizado no ano de 2008. Este censo evidenciou 31.922 pessoas adultas vivendo em situação de rua no país ${ }^{2}$. Entretanto, sabe-se que esta população vem crescendo no país, e esta realidade reproduz a invisibilidade social desta população no âmbito das políticas públicas. Algumas situações relacionadas ao contexto de vida nas ruas são compreendidas pelo imaginário popular como inerentes a este cenário, contudo estas estão estreitamente associadas às dissimetrias que caracterizam as sociedades desiguais ${ }^{3}$.

A realidade de quem vive nas ruas é marcada desta forma pela exclusão social e política, que resulta na privação de direitos constitucionais básicos, além da vulnerabilidade extrema e sofrimentos. Neste sentido, vários direitos são negados, tais como o exercício da cidadania, o acesso à saúde, trabalho e habitação, além da exposição às condições precárias de sobrevivência e outras diversas formas de violência. Estas vulnerabilidades e comportamentos de risco vivenciados pela população em situação de rua culminam no comprometimento da saúde física e mental dessa população $0^{4,5}$.

O aumento do número de pessoas em situação de rua pode estar diretamente relacionado às iniquidades e desigualdades, concentrando o maior contingente nas grandes metrópoles. As ruas muitas vezes são buscadas como meio de sobrevivência e falta de habitação, porém outras motivações estão envolvidas, como o rompimento de vínculos familiares e utilização deste espaço para o consumo de drogas, isento de controles ou limites de terceiros6.

Apesar de que pessoas em situação de rua possam partilhar características em comum, cada cidade tem características sociais, econômicas, políticas e culturais que a diferem uma das outras, produzindo singularidades nesta população na medida em que habitam o contexto urbano. Portanto, as especificidades deste grupo devem ser consideradas, tais como: gênero, raça/cor, idade, abuso de drogas e, sobretudo, doenças mentais. Estudos apontam a existência da conexão entre distúrbios mentais e as reações psicológicas anormais que apresentam grande prevalência nos grupos transitórios e situacionais dos moradores de rua ${ }^{1,4,7}$

Neste sentido, é necessário que a rede de atenção à saúde esteja fortalecida e preparada para atuar junto a essa população, portanto informações atualizadas em relação à saúde mental, comportamentos e vulnerabilidades no contexto de vida deste grupo são essenciais para o planejamento de ações de acordo com as suas especificidades. Deste modo, considerando a necessidade do fortalecimento da Rede de Atenção Psicossocial (RAPS), e a escassez de informações epidemiológicas recentes em relação à saúde mental da população de rua, esse estudo tem como objetivo analisar a saúde mental de pessoas em situação de rua, comportamentos e vulnerabilidades no contexto urbano.

\section{MÉTODO}

Estudo exploratório, transversal, analítico, com abordagem quantitativa, realizado na cidade do Recife, capital do estado de Pernambuco. A população do estudo foi constituída pelos indivíduos adultos em situação de rua, de ambos os sexos, com idade igual ou superior a 18 anos. Os participantes que não conseguiram compreender e/ou responder os instrumentos da pesquisa compuseram o critério de exclusão.

A amostra foi calculada de forma estratificada proporcional, com base no número de pessoas que foram contabilizadas pelo mapeamento de pessoas em situação de rua realizada no ano de 2014 pela Secretaria de Desenvolvimento Social e Direitos Humanos da prefeitura da cidade do Recife 8 .

O tamanho amostral foi determinado considerando-se: o objetivo de determinação dos percentuais das variáveis dependentes; a margem de erro de 5,0\%; confiabilidade de $95 \%$ de que a margem não seja ultrapassada; tama- 
nho populacional de 719 moradores de rua; prevalência esperada de $50 \%$, valor que maximiza o tamanho amostral. Assim, o tamanho amostral necessário foi 251 pesquisados. Foi acrescido o estudo piloto com 23 participantes realizado para verificação e adequação dos instrumentos do estudo garantindo uma margem de segurança para perdas eventuais. A amostra foi distribuída proporcionalmente entre os distritos sanitários da Prefeitura da Cidade do Recife, de acordo com a contagem de cada distrito. O cálculo foi realizado por meio do programa MINITABß versão 17.

A coleta foi realizada entre julho de 2015 a julho de 2016. O tempo médio de cada entrevista variou entre $20 \mathrm{e}$ 30 minutos. Os usuários que estavam sob efeito de drogas no primeiro contato, foram abordados em outro momento da continuidade da coleta de dados. Os retornos ao campo para continuar a entrevista foram essenciais para evitar perdas, retomando a coleta de dados quando não foi possível abranger um maior número de pessoas.

As variáveis sociodemográficas foram avaliadas pelo instrumento de entrevista semiestruturado, construído pelos pesquisadores para finalidade da pesquisa, dividido por quatro blocos de identificação das variáveis contendo: dados sociodemográficos, condição de vida nas ruas, situação atual, histórico de saúde e de consumo de drogas. Pela característica do estudo, as variáveis foram autodeclaradas pelos participantes e categorizadas posteriormente.

Para avaliar transtornos mentais foi utilizado o instrumento International Neuropsychiatric Interview (MINI Plus 5.0.0) para saúde mental. Este instrumento consiste em uma entrevista diagnóstica estruturada que investiga os transtornos mentais do eixo I do Diagnostic and Statistical Manual of Mental Disorders (DSM V) e da Classificação Internacional de Doenças (CID 10) DSM-V-R e da CID-10 (AMORIM, 2000)9.

O teste de triagem para o consumo de Substâncias Psicoativas (SPA) foi utilizado apenas para os entrevistados que responderam positivamente ao critério de uso abusivo de (SPA). Foi utilizado o Alcohol, Smoking and Substance Involvement Sreenning Test (ASSIST), traduzido e validado para uso no Brasil em 2004. Este instrumento é formado por um questionário estruturado que investiga nove tipos de drogas. O resultado do ASSIST permite classificar o padrão do uso, facilitando os procedimentos de intervenção. As intervenções indicadas pelo ASSIST são intervenção breve e tratamento intensivo. Na intervenção breve o acompanhamento deve ser realizado pelo menos uma vez por semana, e menos de três dias, já no tratamento intensivo a intervenção é mais aprofundada, necessitando mais do que três dias seguidos ${ }^{10}$.

Os dados foram digitados em dupla entrada em planilhas EXCEL $® 2013$ e analisados descritivamente por meio da distribuição de frequências absolutas e relativas por meio do programa Epilnfo®. A execução deste estudo obedeceu aos preceitos éticos exigidos, em consonância com as etapas estabelecidas pela Resolução n066/12 do Conselho Nacional de Saúde que trata sobre os aspectos éticos com pesquisa envolvendo seres humanos. $O$ estudo conta com o parecer de número 1.059.357 do Comitê de Ética em Pesquisa da Universidade Federal de Pernambuco.

\section{RESULTADOS}

Participaram do estudo 274 pessoas. A caracterização do perfil sociodemográfico da amostra apresentou predomínio do sexo masculino $(52,9 \%)$, a faixa etária entre 30 e 39 anos (31,8\%), com ensino fundamental incompleto $(51,2 \%)$, solteiro $(60,6 \%)$ e sem religião $(38,7 \%)$. 
TABELA 1. Distribuição do perfil sociodemográfico da população de rua, residentes da cidade do Recife-PE, 2016.

\begin{tabular}{|c|c|c|}
\hline Variáveis & $\begin{array}{l}\text { Frequência } \\
\text { (n) }\end{array}$ & $\begin{array}{c}\text { Percentual } \\
(\%)\end{array}$ \\
\hline \multicolumn{3}{|l|}{ Sexo } \\
\hline Masculino & 144 & 52,9 \\
\hline Feminino & 130 & 47,1 \\
\hline \multicolumn{3}{|l|}{ Faixa etária } \\
\hline 18 a 19 anos & 9 & 3,6 \\
\hline 20 a 29 anos & 57 & 20,9 \\
\hline 30 a 39 anos & 88 & 31,8 \\
\hline 40 a 49 anos & 56 & 20,4 \\
\hline 50 a 59 anos & 36 & 13,1 \\
\hline 60 ou mais & 28 & 10,2 \\
\hline \multicolumn{3}{|l|}{ Anos de estudo } \\
\hline Não alfabetizado & 45 & 16,4 \\
\hline Fundamental incompleto & 140 & 51,2 \\
\hline Fundamental completo & 29 & 10,6 \\
\hline Ensino médio incompleto & 28 & 10,2 \\
\hline Ensino médio completo & 27 & 9,9 \\
\hline Superior incompleto & 1 & 0,2 \\
\hline Superior completo & 4 & 1,5 \\
\hline \multicolumn{3}{|l|}{ Estado conjugal } \\
\hline Solteiro & 166 & 60,6 \\
\hline Casado/União estável & 68 & 24,8 \\
\hline Divorciado/separado & 14 & 5,1 \\
\hline Viúvo & 22 & 8,0 \\
\hline Outros & 4 & 1,5 \\
\hline \multicolumn{3}{|l|}{ Religião } \\
\hline Não possui & 106 & 38,7 \\
\hline Católicos & 84 & 30,7 \\
\hline Evangélicos & 57 & 20,8 \\
\hline Espíritas & 17 & 6,2 \\
\hline Cristãos & 8 & 2,9 \\
\hline Umbanda & 2 & 0,7 \\
\hline Total & 274 & 100 \\
\hline
\end{tabular}

Fonte: Autoria própria

A partir da aplicação do ASSIST foi identificado que 175 participantes apresentaram um padrão de uso abusivo de drogas, dos quais (20\%) apresentaram indicação de tratamento intensivo para o uso do álcool, seguido de $(38,9 \%)$ de intervenção breve para esta substância. Para o uso do crack o percentual de $(16 \%)$ dos indivíduos foi indicado para tratamento intensivo e (13,7\%) para intervenção breve. A utilização da maconha pelos participantes 
apresentou o percentual de $(12,6 \%)$ para o tratamento intensivo e $38,3 \%$ para intervenção breve.

TABELA 2. Avaliação do ASSIST para uso de drogas pela população de rua da cidade do Recife-PE, 2016. $\mathrm{n}=175$

\begin{tabular}{|c|c|c|c|c|c|c|}
\hline \multirow{2}{*}{ Drogas } & \multicolumn{2}{|c|}{ Nenhuma intervenção } & \multicolumn{2}{|c|}{ Intervenção breve } & \multicolumn{2}{|c|}{ Tratamento intensivo } \\
\hline & $\mathrm{N}$ & $\%$ & $\mathbf{N}$ & $\%$ & $\mathbf{n}$ & $\%$ \\
\hline Tabaco & 89 & 50,9 & 68 & 38,9 & 18 & 10,3 \\
\hline Álcool & 79 & 45,1 & 60 & 34,3 & 35 & 20,0 \\
\hline Maconha & 86 & 49,1 & 67 & 38,3 & 22 & 12,6 \\
\hline Anfetaminas & 175 & 100,0 & - & - & - & - \\
\hline Inalantes & 146 & 83,4 & 11 & 6,3 & 18 & 10,3 \\
\hline Cocaína & 164 & 93,7 & 8 & 4,6 & 3 & 1,7 \\
\hline Hipnóticos/sedativos & 175 & 100,0 & - & - & - & - \\
\hline Alucinógenos & 174 & 99,4 & - & - & - & - \\
\hline Opioides & 175 & 100,0 & - & - & - & - \\
\hline Crack & 125 & 71,4 & 24 & 13,7 & 28 & 16,0 \\
\hline Calmante & 170 & 97,1 & 2 & 1,1 & 3 & 1,7 \\
\hline
\end{tabular}

Fonte: Autoria própria, 2016.

A tabela três apresenta os resultados relacionados à aplicação do MINI em todos os entrevistados. A dependência/abuso de substância não alcoólica apresentou prevalência em $(40,9 \%)$, seguido do transtorno de personalidade $(24,8 \%)$ e do episódio depressivo maior $(22,6 \%)$. O transtorno de estresse pós-traumático se apresentou em $(5,1 \%)$ da população, seguido do transtorno de ansiedade generalizada $(2,9 \%)$ e da distimia $(1,8 \%)$. $O$ risco de suicídio atual foi evidenciado em $(38,3 \%)$ dos entrevistados.

TABELA 3. Resultado da aplicação do MINI aplicado na população de rua, residentes da cidade do Recife-PE, 2016.

\begin{tabular}{lrc}
\hline \multicolumn{1}{c}{ Resultados do MINI } & Frequência & Percentual \\
\hline \multicolumn{1}{c}{ Variável } & $\mathbf{n}$ & $\%$ \\
\hline Episódio depressivo maior & 62 & 22,6 \\
Distimia & 5 & 1,8 \\
Risco de suicídio atual & 105 & 38,3 \\
Transtorno de estresse pós-traumático & 14 & 5,1 \\
Dependência / Abuso substância não alcoólica & 112 & 40,9 \\
Transtorno de ansiedade generalizada & 8 & 2,9 \\
Transtorno de personalidade generalizada & 68 & 24,8
\end{tabular}

Fonte: Autoria própria, 2016.

Na tabela 4 que avalia o comportamento e vulnerabilidade da população em situação de rua, foi verificado que a maior parte dos entrevistados referiu ausência de transtornos mentais na família (43,1\%). Mais da metade da população entrevistada afirmou ter algum problema de saúde com prevalência para os problemas psicológicos, mentais ou neurológicos $(39,6)$. Durante o período de um ano, $(66,4 \%)$ da população referiu que raramente ou nunca buscaram atendimento na RAPS. A maioria dos participantes afirmou ter sofrido algum tipo de violência, sendo a agressão física a causa mais prevalente $(42,4 \%)$. 
TABELA 4. Informações sobre o comportamento e vulnerabilidades em saúde da população de rua, residentes da cidade do Recife-PE, 2016.

\begin{tabular}{|c|c|c|}
\hline \multicolumn{3}{|c|}{ COMPORTAMENTO E VULNERABILIDADES EM SAÚDE NAS RUAS } \\
\hline Realiza higiene diária & Frequência (n) & Percentual (\%) \\
\hline Sim & 228 & 83,2 \\
\hline Não & 46 & 16,8 \\
\hline Total & 274 & 100 \\
\hline Doença mental na família & Frequência (n) & Percentual (\%) \\
\hline Sim & 108 & 39,4 \\
\hline Não & 118 & 43,1 \\
\hline Não sabe & 48 & 17,5 \\
\hline Total & 274 & 100 \\
\hline Problemas de saúde referidos & Frequência (n) & Percentual (\%) \\
\hline Psicológicos, mentais ou neurológicos & 61 & 39,6 \\
\hline IST's & 36 & 23,4 \\
\hline Respiratório & 27 & 17,5 \\
\hline HAS & 21 & 13,6 \\
\hline Diabetes & 15 & 9,7 \\
\hline Pele & 18 & 11,7 \\
\hline Outro & 46 & 29,9 \\
\hline Total & 154 & - \\
\hline Frequência da Procura de atendimento de saúde por ano & Frequência (n) & Percentual (\%) \\
\hline Raramente / Nunca & 182 & 66,4 \\
\hline 1 a 3 vezes & 36 & 25,2 \\
\hline Mais de 3 vezes & 21 & 7,7 \\
\hline Não informado & 02 & 0,7 \\
\hline Local de atendimento & Frequência (n) & Percentual (\%) \\
\hline ESF & 45 & 16,4 \\
\hline UPAS & 36 & 13,1 \\
\hline Policlínicas & 27 & 9,9 \\
\hline CnaR e CR & 20 & 7,3 \\
\hline CAPS & 8 & 2,9 \\
\hline Outros & 17 & 6,2 \\
\hline Não informado & 121 & 44,2 \\
\hline Total & 274 & 100 \\
\hline Violência nas ruas & Frequência (n) & Percentual (\%) \\
\hline Agressão física & 116 & 42,4 \\
\hline Não sofreu & 96 & 35,0 \\
\hline Violência sexual & 34 & 12,3 \\
\hline Agressão verbal & 18 & 6,6 \\
\hline Roubo & 6 & 2,2 \\
\hline Maus tratos & 4 & 1,5 \\
\hline Total & 274 & 100 \\
\hline
\end{tabular}

Fonte: Autoria própria, 2016. 


\section{DISCUSSÃo}

Pesquisas realizadas com a população em situação de rua descrevem predomínio do sexo masculino entre esses indivíduos ${ }^{11-14}$. Entretanto, nesse estudo, verificaram-se proporções semelhantes em relação ao sexo. De modo geral, as mulheres são mais receptivas e abertas ao diálogo, o que pode ter contribuído para a homogeneidade apresentada nesta variável, uma vez que as entrevistas neste estudo foram realizadas in loco.

No tocante à faixa etária, achados semelhantes foram verificados em pesquisa realizada nas cidades de Ottawa, Toronto e Vancouver, em que os entrevistados apresentaram idade entre 30 e 39 anos. Os resultados revelam que a maioria das pessoas que moram nas ruas encontra-se em idade produtiva ${ }^{12,14,15}$.

Apesar de que grande parte declarou estar solteira e sem companheiros fixos, vivem em companhia de colegas de rua ou com entes familiares. Estes dados corroboram com pesquisa realizada na zona central de uma capital do nordeste brasileiro, em que $84 \%$ dos pesquisados se declararam solteiros 16 . Apesar disso, observa-se que, independente de relações conjugais, vínculos afetivos são estabelecidos nestes cenários, e mesmo que ainda inconstantes, podem ser considerados como rede de proteção e apoio para o enfrentamento da vida nas ruas ${ }^{17}$.

A baixa escolaridade verificada nesta população pode possuir forte relação com as escassas oportunidades dessas pessoas devido ao seu histórico de extrema pobreza e negligência social ${ }^{16}$. A menor quantidade de anos estudados se reflete também no acesso e procura aos serviços de saúde, uma vez que pode influenciar o seu conhecimento e percepção sobre o próprio cuidado.

O consumo de drogas se apresenta como um fenômeno presente neste modo de vida que inclui processos adaptativos e relações sociais neste meio, quer seja pelo uso individual, ou em grupos, no sentido de que muitas vezes buscam aliviar as adversidades ocasionadas pela vida nas ruas. Corroborando com esta linha de pensamento, pesquisadores avaliam que existe uma funcionalidade relacionada ao uso do álcool, pelo favorecimento de encontros coletivos, além de anestesiar o sofrimento causado por viver em situação de rua ${ }^{18,19}$. Outro estudo realizado em Pernambuco revelou que moradores de rua muitas vezes utilizam o álcool como terapia, além da busca de inserção neste modo de vida ${ }^{20}$

Problemas legais relacionados ao uso de drogas foram relatados, podendo estar diretamente associados ao envolvimento em atos ilícitos motivados pelo uso destas substâncias, que por outro lado, podem ser produtores de violência, envolvendo agressões, ações policiais, ou até mesmo prisões. Estudo multicêntrico realizado em seis capitais brasileiras relatou que existe um grande envolvimento relacionando questões legais com o uso do crack, por esta droga ter uma intrínseca relação com a violência ${ }^{21}$.

A maior parte dos entrevistados referiu consumo frequente de drogas, destacando-se o uso de álcool entre as outras drogas. Muitos referiram o uso antes mesmo de irem para as ruas, e outros passaram a fazê-lo depois. Logo, se compreende que existem questões relativas à realidade de cada indivíduo e sua relação com as drogas, bem como o viver nas ruas. Pesquisa realizada em Madrid, na Espanha, também mostrou que o álcool é a droga de maior prevalência na análise dos padrões de consumo da população de rua no país ${ }^{21,22}$.

O consumo do crack com padrão de uso diário foi referido por pouco mais da metade dos entrevistados neste estudo. Apesar de o crack ser uma droga relativamente recente, observa-se que a utilização desta droga vem crescendo entre a população de rua ${ }^{22}$. Este aspecto chama à atenção para o fato de que o crack tenha uma inserção maior nessas populações com maior vulnerabilidade social, provavelmente pelo baixo custo da droga e o intenso e rápido efeito que ela proporciona ${ }^{23}$.

Outro dado relevante com relação a essa droga é uma maior prevalência de sintomas depressivos encontrados nos usuários moradores de rua, somada a um maior risco de cometer suicídio ${ }^{24}$. Neste estudo, verificou-se ocorrência de episódio depressivo nos participantes com associação ao consumo mais elevado de crack. Um estudo sociodemográfico sobre usuários de crack em Minas Gerais verificou que 37,5\% apresentaram transtornos mentais e comportamentais associados ao uso de outras drogas, enquanto $62,5 \%$ apresentaram comorbidades psiquiátricas ${ }^{18}$.

O abuso de drogas não foi reconhecido como um problema de saúde pela maioria dos participantes, entretanto o resultado do MINI apontou que quase metade destes apresentaram dependência ou abuso de substância não alcoólica, que se enquadram nos transtornos relacionados às substâncias pelo DSM V9. O ASSIST, por sua vez, 
apontou necessidade de tratamento intensivo para abusadores de álcool, crack, maconha, tabaco e inalantes (DSM V, 2013) ${ }^{10}$. Pela análise psicossocial, esse fenômeno pode ser explicado diante do reconhecimento das causas que os levaram a usar drogas, pois muitos relatam ser um meio de refúgio, bem como uma manobra de enfrentamento, valendo salientar que alguns as consideram imprescindíveis em seu cotidiano nas ruas ${ }^{24}$.

Estas questões, apesar de dificultarem a adesão ao tratamento, necessitam também serem analisadas considerando a subjetividade destas pessoas que apresentam diferentes sistemas de crenças, valores e experiências que validam a compreensão do seu processo saúde-doença e o lugar da droga no seu modo de vida ${ }^{25,26}$.

Dentre os diagnósticos identificados pelo MINI, foi constatado que o transtorno de personalidade generalizada é um dado significativo na população de rua, podendo estar relacionado ao uso de drogas, e ou o envolvimento com atos ilícitos, bem como outras formas de violação de direitos. Os transtornos de personalidade sofrem influências, tanto biológicas como do ambiente, portanto algumas pessoas adquirem hábitos e atitudes caracterizadas por falta de resiliência, que se vão transformando a experiência de vida dessas pessoas ${ }^{27}$.

Pesquisa realizada em Belo Horizonte destacou que existe carência de estudos no Brasil que se aprofundem acerca dos transtornos de personalidade em moradores de rua. Assim, os resultados desta pesquisa indicaram que o problema mental relacionado a desvios de personalidade pode ser mais proeminente em moradores de rua, demandando uma maior atenção e cuidado com a saúde mental desse público ${ }^{28}$.

A ocorrência da doença depressiva avaliada neste estudo apresentou menor prevalência do que uma pesquisa anterior realizada no Brasil, que detectou o percentual de $56,3 \%$ de depressão maior em homens moradores de rua29. Os sintomas depressivos envolvem diversas alterações cognitivas, motoras e somáticas que interferem no funcionamento do indivíduo em diversas áreas, e ainda podem ser agravadas por características evidenciadas nos participantes deste estudo, tais como: ausência de crenças religiosas, baixa autoestima e desesperança ${ }^{30}$.

O risco de suicídio atual é um dado relevante neste estudo. Para além da questão de segurança, o suicídio é um problema grave de saúde pública que demanda intervenção imediata, destacando-se os graus identificados para risco de vida iminente. Pesquisa realizada no Japão investigou a correlação de comportamento suicida na população de rua e relatou que a falta de suporte social, a percepção subjetiva de saúde, deficiência visual, dor, insônia, mal-estar mental e depressão atual foram significativamente associados aos pensamentos recorrentes de suicídio ${ }^{31}$.

Vale ressaltar que alguns estudos também relacionam o aumento de pessoas com doenças mentais nas ruas com o processo de desinstitucionalização, além de taxas de mortalidade elevadas entre estes indivíduos ${ }^{32,33}$. Em Maryland, nos EUA, pesquisa sobre causas de morte violenta em moradores de rua, reportou que $65,2 \%$ das mortes foram classificadas como indeterminadas, que o uso e abuso de drogas foram comuns no momento das mortes, e que estas pessoas não estavam em tratamento ${ }^{33}$.

Assim, torna-se evidente a existência de um ciclo que envolve a doença mental, o morador de rua e o uso e abuso de drogas, em que os determinantes sociais contribuem para o processo de adoecimento, tornando esta problemática um desafio que requer desdobramentos políticos intersetoriais ${ }^{34}$.

A exclusão social, vivenciada de forma histórica pela população em situação de rua, se agrava pelos abusos e crimes realizados a esta população vulnerável ${ }^{21}$. Essa vulnerabilidade presente no cotidiano desses indivíduos pode ser observada por meio da violência nas ruas ${ }^{27}$. $O$ índice de violência contra essas pessoas cresce continuamente. Uma pesquisa realizada no Rio de Janeiro relatou que entre os meses de março e julho de 2017, houve aumento de $60 \%$ nas denúncias relacionadas ao constrangimento e violência contra moradores de rua ${ }^{30}$. Neste estudo, foi evidenciado que a maior parte dos entrevistados relatou já ter sofrido algum tipo de violência, dentre estas, as agressões físicas apresentaram destaque.

As pessoas que vivenciam a situação de rua também sofrem outros tipos de violência, e em outros locais fora da rua, como por exemplo, nos serviços de saúde. São geralmente vítimas de preconceitos e despreparo dos profissionais no atendimento às suas necessidades e especificidades. Portanto, a maior parte dessas pessoas não procuram os serviços de assistência à saúde, apesar de relatarem enfermidades. Mesmo diante deste reconhecimento, as condições de saúde não limitam o trabalho, pois são relativizadas frente à necessidade de sobrevivência. Ter saúde, na concepção de um indivíduo em situação de rua, é estar vivo e resistir às dificuldades diárias, é poder se locomover 5 .

DOI: $10.33947 / 1982-3282-v 15 n 3-4-4667$ 
Neste sentido, o acesso à saúde para o morador de rua é uma ferramenta imprescindível para ofertar cuidados de saúde física e mental equânimes, entretanto, a dificuldade de acesso não deve ser compreendida como negligência à saúde por parte do morador de rua. Além disso, torna-se necessária a compreensão de que a doença é uma construção social e, parece ser peculiar nesta população que a consciência dos agravos à sua saúde fique em segundo plano em relação à preocupação em conseguir alimento, abrigo seguro e alguma fonte de renda para sobreviverem.

Diante de tal realidade, é imperioso garantir que os serviços de saúde funcionem de forma articulada e que haja investimento nos dispositivos especializados para o acesso à saúde deste grupo. Verifica-se que esta população normalmente se reporta aos serviços de saúde em situações emergenciais, que aparecem como principal via de contato com os serviços de saúde, talvez por reconhecerem o corpo doente quando os sintomas se intensificam, ou pela incapacidade de se locomoverem, ou ainda, devido a episódios de mau atendimento ou impedimento nas unidades de saúde.

Este estudo apresentou limitações importantes, pois foi coletado no contexto de vida nas ruas, havendo interferência de condições climáticas e demandou tempo maior para a formação do vínculo antes que as entrevistas acontecessem. Além disso, todas as informações foram autodeclaradas pelos participantes. Apesar das adversidades vivenciadas pela população de rua, após se sentirem confiantes, estes se mostraram disponíveis para serem acolhidos, demonstrando a necessidade de atenção e respeito que esse público necessita.

\section{CONCLUSÃO}

O viver nas ruas pode afetar a saúde física e mental, não somente pelas condições de vida nestes espaços, como também pelo comportamento de risco para saúde. O abuso de drogas, entre outros comportamentos de risco, contribui para que a saúde física e mental da população de rua represente um desafio constante, estando relacionado com a dependência química, aumento da mortalidade, transtorno depressivo e risco aumentado para o suicídio nesta população. Este estudo evidencia que as condições de vida no cenário das ruas se mantêm insalubres e ainda distantes de políticas públicas efetivas.

Concluímos que a saúde mental da população de rua se apresenta fragilizada diante do contexto que inclui o prejuízo do atendimento às necessidades básicas, da falta de acesso aos serviços de saúde e dos comportamentos de risco rotineiros do qual fazem parte da vida nas ruas, entre estes, ausência de tratamento para os transtornos mentais e para o abuso de drogas. Apesar desta ser uma realidade conhecida, parece ser ignorada pela sociedade e pelo poder público. Portanto, a atualização de estudos epidemiológicos sobre os agravos a essa população se faz necessários, pois problemas conhecidos que se perpetuam representam importantes lacunas no conhecimento para ações efetivas.

O fortalecimento da RAPS, sobretudo os serviços de referência para população em situação de rua devem ser fortalecidos, com equipes multidisciplinares, favorecendo os múltiplos olhares para organização de fluxos que acolham as especificidades desta população nos moldes equânimes e integrais.

\section{REFERÊNCIAS}

1. Silva FPD, Silva ECD, Ferreira AL, Frazão IDS. Segurança do paciente: ao alcance das pessoas em situação de rua? Revista Brasileira de Enfermagem. 2020;73(5):1-8.

2. Brasil, Ministério do Desenvolvimento Social e Combate à Fome. Secretaria de Avaliação e Gestão da Informação. Pesquisa Nacional sobre a População em Situação de Rua. Brasília: MDS, 2008 [Internet]. Brasil: Meta Instituto de Pesquisa de Opinião; 2008 [cited 2020 Maio 6]. Available from: https://www.mds.gov.br/webarquivos/publicacao/ assistencia_social/Livros/Rua_aprendendo_a_contar.pdf

3. Brasil, Prefeitura de São Paulo divulga Censo da População em Situação de Rua 2019 [Internet]. São Paulo; 2020 Jan 31 [acesso em: 2020 Maio 5]. Disponível em: http://www.capital.sp.gov.br/noticia/prefeitura-de-sao-paulo- 
-divulga-censo-da-populacao-em-situacao-de-rua-2019

4. Silva SYS, Santos Rosa LC, Lima MDCS. Perfil das pessoas em situação de fragilidade social atendidas pelo "consultório na rua" na cidade de teresina-pi. Revista Ciência Plural. 2020;108-19.

5. Ferreira CPDS, Rozendo CA, Melo, GBD. Consultório na Rua em uma capital do Nordeste brasileiro: o olhar de pessoas em situação de vulnerabilidade social. Cadernos de Saúde Pública. 2016; 32(1):e00070515.

6. Hino P, Santos JDO, Rosa ADS. Pessoas que vivenciam situação de rua sob o olhar da saúde. Revista Brasileira de Enfermagem. 2018;71(1): 684-92.

7. Brandão MGSA, Aragão CP, Ximenes MAM, Caetano JÁ. Autoconceito, depressão e ansiedade de pessoas em situação de rua. Revista Enfermagem Atual In Derme. 2019;90(28):1-6.

8. Secretaria de Desenvolvimento Social e Direitos Humanos. Contagem de pessoas em situação de rua no Município do Recife. Recife; 2014.

9. Amorim P. Mini International Neuropsychiatric Interview (MINI): validação de entrevista breve para diagnóstico de transtornos mentais. Revista Brasileira de Psiquiatria, Rio de Janeiro. 2000;22(3):106-15.

10. Henrique IFS. Validação da versão brasileira do teste de triagem do envolvimento com álcool, cigarro e outras substâncias (ASSIST). Revista da Associação Médica Brasileira, São Paulo. 2004;50(2):199-206.

11. Silva GJ, Ximenes MAM, Brandão MGSA, Brito ÓD, Barros LM. Aplicação do modelo de Roper, Logan e Tierney com pessoas em situação de rua. Revista de Pesquisa: Cuidado é Fundamental. 2020;238-45.

12. Patrício ACFDA, Silva RARD, Araújo RFD, Silva RFD, Nascimento GTDS, Rodrigues TDDB et al. Transtornos mentais comuns e resiliência de pessoas em situação de rua. Revista Brasileira de Enfermagem. 2019;72(6):1526-33.

13. Yamamoto M, Watanabe T, Uehara R, Horita R, Sado T, Nishio A. Prevalence of diabetes among homeless men in Nagoya, Japan: A survey study. Journal of Diabetes Investigation. 2019;10(3): 667-72.

14. Landefeld JC, Miaskowski C, Tieu L, Ponath C, Lee CT, Guzman D, et al. Characteristics And Factors Associated With Pain In Older Homeless Individuals: Results From The Health Outcomes In People Experiencing Homelessness In Older Middle Age (HOPE HOME) study. The Journal of Pain. 2017;18(9):1036-45.

15. Duhoux A, Aubry T, Ecker J, Cherner R, Agha A, To MJ, Palepu A. Determinants of Unmet Mental Healthcare Needs of Single Adults Who Are Homeless or Vulnerably Housed. Canadian Journal of Community Mental Health. 2018;36(3):41-57.

16. Silva EF, Brito GMI, Oliveira VMC, Carvalho MSM, Sousa BBV, Magalhães RDLB. Conhecimento sobre o HIV/ Aids de pessoas em situação de rua. Revista Eletrônica Acervo Saúde. 2019;(27):e836.

17. Sicari AA, Zanella AV. Pessoas em situação de rua no Brasil: revisão sistemática. Psicologia: Ciência e Profissão. 2018;38(4):662-79.

18. Mendes KT, Ronzani TM, Paiva FSD. População em situação de rua, vulnerabilidades e drogas: uma revisão sistemática. Psicologia \& Sociedade. 2019;31. 
19. Peiter P, Belmonte P, Teixeira M, Gomes MF, Lacerda A. Territorialidade Dos Usuários De Crack Em Situação De Rua E Suas Redes De Apoio Social No Município Do Rio De Janeiro. Hygeia-Revista Brasileira De Geografia Médica E Da Saúde. 2017;13(26):192-97.

20. Silva FP, Paiva FAS, Guedes CP, Frazão IS, Vasconcelos SC, Lima MDC. Nursing Diagnoses of the Homeless Population in Light of Self-care Theory. Archives of psychiatric nursing. 2018;32(3):425-31.

21. Teixeira MB, Belmonte P, Engstrom NEM, Lacerda A. Os invisibilizados da cidade: o estigma da População em Situação de Rua no Rio de Janeiro. Saúde em Debate. 2020;43:92-101.

22. Seleghim MR, Galera SAF. Qual a relação do uso de crack com a ocorrência da situação de rua?. Saúde \& Transformação Social/Health \& Social Change. 2017;8(1):01-08.

23. Zeferino MT, Fermo VC, Fialho MB, Kenthi A, Bastos FI. Cenas De Uso Do Crack Na Capital De Santa Catarina/ Brasil: A (In) Visibilidade Do Usuário. Texto \& Contexto-Enfermagem. 2019;28.

24. Abreu DD, Oliveira WFD. Atenção à saúde da população em situação de rua: um desafio para o Consultório na Rua e para o Sistema Único de Saúde. Cadernos de Saúde Pública. 2017;33: e00196916.

25. Oliveira LIA, Eduarda RT, Justel PR. Percepções sobre trabalho: análise de concepções de pessoas em situação de rua. Brazilian Journal of Occupational Therapy/Cadernos Brasileiros de Terapia Ocupacional. 2017;25(4).

26. Laporte A, Vandentorren S, Détrez MA, Douay C, Le Strat Y, Le Méner E. Samenta Research Group. Prevalence of mental disorders and addictions among homeless people in the greater Paris area, France. International journal of environmental research and public health. 2018;15(2):241.

27. Araújo AM, Duarte AV, Ribeiro JS, Magalhãe MH, Pontelo I. Ausência de condições de vida da população de rua na região metropolitana de belo horizonte. Revista De Trabalhos Acadêmicos-Universo Belo Horizonte. 2017;1(2).

28. Kim YJ, Boyas JF, Lee KH, Jun JS. Suicidality among homeless people: Testing the mediating effects of self-efficacy and depression. Journal of Human Behavior in the Social Environment. 2019;29(7):922-36.

29. Venturini, E. O espaço e o tempo da desinstitucionalização. Physis: Revista de Saúde Coletiva, 2019;29(4):e290417.

30. Moreira MIB, Guerrero AVP, Bessoni EA. Entre desafios e aberturas possíveis: vida em liberdade no contexto da desinstitucionalização brasileira; 2019.

31. Stanley JL, Jansson AV, Akinyemi AA, Mitchell CS. Characteristics of violent deaths among homeless people in Maryland, 2003-2011. American journal of preventive medicine. 2016;51(5):260-66.

32. Monteiro FKV, Almeida LP. A exclusão social de mulheres moradoras de rua: questões de gênero e políticas sociais. Territorio (s), género, trabajo y políticas públicas en América Latina. São Paulo: Provocare; 2017. p. 117-29.

33. Neves-Silva P, Martins GI, Heller L. “A gente tem acesso de favores, né?”. A percepção de pessoas em situação de rua sobre os direitos humanos à água e ao esgotamento sanitário. Cadernos de Saúde Pública. 2018;34:e00024017.

34. Santos ACED, Brunfentrinker C, Pena LDS, Saraiva SDS, Boing AF. Análise e comparação dos desfechos do tratamento de tuberculose na população em situação de rua e na população geral do Brasil. Jornal Brasileiro de Pneumologia. 2021;47(2):1-4. 